\title{
Was geht schief? Schlichtungsverfahren nach Mammareduktionen - eine Analyse der Eingriffe mit Behandlungsfehler und Haftungsanspruch der Norddeutschen Schlichtungsstelle
}

\section{What went wrong? Analysis of Medical Malpractice Arbitration Proceedings Conducted by a German Arbitration Board after Breast Reductions}

Autoren

Institute

\author{
S. Allert ${ }^{1}$, C. Flechtner ${ }^{2}$, P. M. Vogt ${ }^{3}$, C. Herold
}

${ }^{1}$ Klinik für Plastische und Ästhetische Chirurgie, Handchirurgie, Sana Klinikum Hameln Pyrmont, Hameln ${ }^{2}$ Lungenklinik, Ev. Lungenklinik Berlin Buch, Berlin

${ }^{3}$ Klinik für Plastische, Hand- und Wiederherstellungschirurgie, Zentrum für Schwerbrandverletzte, Medizinische Hochschule Hannover, Hannover

\section{Schlüsselwörter \\ - Norddeutsche Schlichtungsstelle \\ - Brustreduktion \\ - Mammareduktion \\ - Behandlungsfehler}

Key words

- arbitration board

- malpractice

- breast reduction

- reduction mammoplasty

eingereicht $\quad 21.12 .2015$

akzeptiert 17.2.2016

Bibliografie

DOI http://dx.doi.org/

10.1055/s-0042-103586

Handchir Mikrochir Plast Chir

2016; 48: 101-107

(c) Georg Thieme Verlag KG

Stuttgart · New York

ISSN 0722-1819

Korrespondenzadresse

\section{Dr. Sixtus Allert}

Klinik für Plastische und

Ästhetische Chirurgie

Sana Klinikum Hameln Pyrmont

Handchirurgie

St. Maur Platz 11

31785 Hameln

sixtus.allert@sana.de

\section{Zusammenfassung}

$\nabla$

Einleitung: Die Schlichtungsstelle für Arzthaftpflichtfragen der norddeutschen Ärztekammern, kurz: Norddeutsche Schlichtungsstelle ist für insgesamt 10 Bundesländer zuständig (Berlin, Brandenburg, Bremen, Hamburg, Mecklenburg-Vorpommern, Niedersachsen, Sachsen-Anhalt, das Saarland, Schleswig-Holstein und Thüringen) und damit die in Deutschland größte Schlichtungsstelle. Das Datenmaterial der Norddeutschen Schlichtungsstelle bietet u.a. einen Einblick in die Fehlerquellen von Behandlungsabläufen bei Mammareduktionen.

Material und Methodik: Analysiert wurden Antragsschreiben der Patienten, im Auftrag der Schlichtungsstellte von unabhängigen Ärzten erstellte Gutachten und die abschließenden Schlichtungsbescheide der 88 von der Norddeutschen Schlichtungsstelle in den Jahren 2000-2007 geführte Schlichtungsverfahren nach Mammareduktionen. Zusätzlich verstrich eine 5-Jahresfrist, in der ggf. auch juristische Auseinandersetzungen hätten geführt werden können. Diese Daten ermöglichen die Untersuchung jedes Falles aus unterschiedlichen Perspektiven. Für die Auswertungen wurde zudem auf die von der Schlichtungsstelle im MERS-Kodierungssystem (Medical Error Reporting System) eingepflegten statistischen Daten zurückgegriffen.

Ergebnisse: Unter den 88 Mammareduktionen fanden sich 37 Eingriffe mit Haftungsanspruch (FMH). In diesen Fällen wurde eine Kausalität zwischen einem Behandlungsfehler und einem Gesundheitsschaden von der Schlichtungsstelle für gegeben erachtet. Damit liegt die Quote der bestätigten Fehler mit $42 \%$ über der durchschnittlichen Quote von ca. 24\% in den gesamten Schlichtungsverfahren der Norddeutschen Schlichtungsstelle. Die Mehrzahl dieser Fälle war von Gynäkologen operiert worden. In 92\% der Fälle mit Haftungsanspruch lagen nach Auffassung der Gutachter Mängel bei der OP-Planung

\section{Abstract \\ $\nabla$}

Introduction: The Arbitration Board for Medical Liability Issues of the Medical Association of North Germany ("Norddeutsche Schlichtungsstelle") is responsible for 10 federal states in Germany (Berlin, Brandenburg, Bremen, Hamburg, Mecklenburg-Western Pomerania, Lower Saxony, Saarland, Saxony-Anhalt, Schleswig-Holstein and Thuringia) and is the largest arbitration board in Germany. The data available from the Norddeutsche Schlichtungsstelle provides an insight into sources of malpractice during the treatment of reduction mammoplasty.

Material und Methods: We analysed patient request, expert opinions prepared by independent physicians on behalf of the Norddeutsche Schlichtungsstelle and the final verdicts of 88 arbitration proceedings after breast reduction mammoplasties performed between 2000 and 2007. This data allows for each case to be addressed from different viewpoints. Furthermore we analysed the statistical data entered into the Medical Error Reporting System by the arbitration board.

Results: Among the 88 patient requests after reduction mammoplasty, the arbitration board found a causal relationship between damage caused to a patient's health and medical malpractice in 37 cases. Therefore, $42 \%$ of requests resulted in a liability case. This is a higher rate than that of general arbitration proceedings, where only in $24 \%$ of all cases a causal relationship is confirmed by the Norddeutsche Schlichtungsstelle. Most patients were operated on by gynaecologists. In $92 \%$ of liability cases, mistakes happened during the planning and the performance of the surgical procedure, mainly during planning (65\%) and surgical incisions (41\%). The patients mainly complained about scars $(78 \%)$, asymmetry $(68 \%)$ and skin necrosis of the areola (24\%). Financial disadvantage was mentioned less often (46\%) than psychological stress (70\%). 
und -Durchführung vor, insbesondere bei der OP Planung (65\%) und Schnittführung (41\%). Die am häufigsten vorgetragenen Beschwerden waren Narbenbildung (78\%), Asymmetrie (68\%) und MAK Nekrose (24\%). Geltend gemacht wurden seltener finanzielle Schäden (46\%) als psychologische Belastungen (70\%).

Diskussion: Die erhöhte Quote von Haftungsansprüchen könnte darin begründet sein, dass formverändernde Brustoperationen komplexere Behandlungen sind als allgemein angenommen und dass zum Gelingen auch die vermeintlich vernachlässigbare Teilschritte der Behandlung, wie z.B. Operationsplanung und postoperative Nachsorge beitragen. Die besonders hohen Anforderungen an Planung, Durchführung und Nachsorge tragen bei der plastisch-ästhetischen Brustchirurgie zu einer vergleichsweise hohen Fehlerquote bei, wenn nicht sorgsam vorgegangen wird. Zudem ist der Faktor Kommunikation nicht zu unterschätzen.

\section{Einleitung}

Im Jahr 2000 veröffentlichten Kohn et al. in ihrer Arbeit „To Err is Human“ [1], dass jährlich 44000-98000 US-Amerikaner durch medizinische Fehler sterben würden [1]. Die genaue Zahl der in Deutschland auftretenden Behandlungsfehler ist nicht verlässlich festzustellen [2]. Bekannt ist, dass jährlich in Deutschland ca. 40000 Arzthaftungsstreitigkeiten wegen Behandlungsfehlern angestrengt werden. Je ca. 10000 verteilen sich auf die $\mathrm{Zi}$ vilgerichte sowie Gutachterkommissionen und Schlichtungsstellen der Landesärztekammern, die Medizinischen Dienste der Krankenkassen (MDK) und die direkte Verhandlung zwischen Anwalt und Haftpflichtversicherung des Arztes [3,4]. Die Zahl der Haftungsstreitigkeiten, die über die Schlichtungsstellen geführt werden, nimmt zu. So wurden im Jahr 2000 etwa 3800 Anträge in der Nordeutschen Schlichtungstelle eingereicht, im Jahre 2014 etwa 4300 Anträge [5].

Die deutschen Schlichtungsstellen und Gutachterkommissionen wurden aufgrund der zunehmenden Arzthaftpflichtprozesse flächendeckend von den deutschen Ärztekammern eingerichtet. Zielsetzung war und ist es, eine Alternative zu den häufig langwierigen und teuren Zivilprozessen zu bieten. Sie sollen die Durchsetzung berechtigter Schadensersatzforderungen aus fehlerhaften ärztlichen Behandlungen erleichtern und gleichzeitig Ärzte vor unberechtigten Forderungen schützen [6,7]. Auf diesem Weg sollen Konflikte zwischen den Betroffenen gelöst, die Zahl der Zivilprozesse verringert und weniger Strafverfahren gegen Ärzte eingeleitet werden [6].

Die Norddeutsche Schlichtungsstelle (SST), von der die für die vorliegende Arbeit zugrunde gelegten Daten zur Verfügung gestellt wurden, ist für insgesamt 10 Bundesländer zuständig (Berlin, Brandenburg, Bremen, Hamburg, Mecklenburg-Vorpommern, Niedersachsen, Sachsen-Anhalt, das Saarland, Schleswig-Holstein und Thüringen) und damit die in Deutschland größte Schlichtungsstelle [8]. Sie wurde im Jahr 1976 gegründet und wird von den Ärztekammern der genannten Bundesländer getragen. Insgesamt sind in der SST 5 hauptamtliche Juristen sowie über 50 ehrenamtliche Fachärzte tätig, es werden so alle Fachrichtungen abgedeckt.

Ein Schlichtungsverfahren hat 5 Verfahrensabschnitte. Das Verfahren beginnt mit der Antragstellung. Dieser Antrag kann gleichermaßen durch Patienten, Ärzte, Krankenhausträger und deren Haftpflichtversicherer bei den Schlichtungsstellen gestellt werden. Das Verfahren ist freiwillig und wird schriftlich geführt.
Discussion: The higher rate of liability claims may be due to the fact the surgical procedures changing the shape of breasts are more complex than generally expected. Not only the surgery itself, but also the adequate planning and aftercare are of predominant importance for patient satisfaction. All these factors lead to the relatively high rate of medical malpractice in plastic aesthetic breast surgery. Also the communication factor should not be underestimated.

Nachdem ein Antrag eingegangen ist, werden im ersten Schritt die Verfahrensvoraussetzungen geprüft und Schweigepflichtentbindungserklärungen aller am Verfahren beteiligten Personen eingeholt. Im zweiten Schritt klärt die Schlichtungsstelle anhand der Krankenakten den Sachverhalt. Auf dieser Grundlage wird durch einen Juristen sowie einen Facharzt der Schlichtungsstelle ein Gutachtenauftrag in Form eines zu beantwortenden Fragenkataloges formuliert. Kommt es zu einem Schlichtungsverfahren, wird darauf geachtet, dass die Begutachtung möglichst fachgleich erfolgt, d.h. in einem Fall, der einen Plastischen Chirurgen betrifft, wird als Gutachter möglichst auch ein Plastischer Chirurg bestellt. Fällt die Behandlung unter einen anderen Facharztstandard, werden Gutachter aus dem entsprechenden Fach ausgewählt. Als Gutachter fungieren in den meisten Fällen externe, nicht an die Schlichtungsstelle gebundene, und aufgrund ihrer Qualifizierung und Subspezialisierung ausgewählte Fachärzte. Die Erstellung des Gutachtens ist der dritte Verfahrensschritt. Bevor der Gutachtenauftrag dem externen Gutachter zugeleitet wird, erhalten alle am Verfahren Beteiligte die Möglichkeit, zum Gutachtenauftrag Stellung zu nehmen. Das fertige Gutachten wird stets den Parteien zur Stellungnahme überlassen. Bestehen begründete Einwände gegen das Gutachten, kann es zu einer erneuten Begutachtung kommen [7]. Viertens wird die Haftungsfrage geklärt. Hierfür wird das Gutachten von den Juristen sowie den ärztlichen Mitgliedern der Schlichtungsstelle geprüft. Stellungnahmen der Parteien zum Gutachten werden berücksichtigt. Die Schlichtungsstelle ist an das Urteil des externen Gutachters nicht gebunden. Sie gibt nach Prüfung des Gutachtens eine abschließende, eigene Stellungnahme zu der fraglichen Haftungsverpflichtung im Rahmen des Schlichtungsbescheides ab. Werden die Ansprüche des Patienten bejaht, werden die Regulierungsverhandlungen über die Höhe der zu zahlenden Schadensersatzansprüche direkt zwischen dem Patienten oder dessen Vertretern und den Haftpflichtversicherern geführt. Zu einem fünften Verfahrensabschnitt, einer erneuten Prüfung, kann es kommen, wenn von den Parteien neue Tatsachen vorgebracht werden, die noch nicht Gegenstand der ersten Prüfung waren [9].

Rund ein Viertel aller Arzthaftungsstreitigkeiten werden in Deutschland heute durch die Schlichtungsstellen und Gutachterkommissionen außergerichtlich entschieden. In 90\% der Fälle werden die Entscheidungen der Stellen von den Parteien auch akzeptiert, obgleich das Urteil nicht rechtlich bindend ist [8]. Da es sich um ein freiwilliges Verfahren handelt, kann ein Schlich- 
tungsverfahren jederzeit beendet werden, auch ist es den Parteien jederzeit möglich, den ordentlichen Rechtsweg einzuschlagen [7].

Haftungsansprüche gegenüber einem Arzt können nur geltend gemacht werden, wenn im Schlichtungsverfahren die Kausalität zwischen einem Behandlungsfehler und Gesundheitsschaden bestätigt wird. Diese Fälle werden im Medical Error Reporting System (MERS) der Schlichtungsstelle unter der Kategorie $10=$ „Fehler mit Schaden“ geführt. In dieser Arbeit werden diese Fälle als „Fälle mit Haftungsanspruch“ (FMH) bezeichnet.

Diese „Fälle mit Haftungsanspruch“ wurden hinsichtlich ihrer Behandlungsmängel bei Mammareduktionen untersucht. Es soll auf Fehlerquellen bei Mammareduktionen aufmerksam gemacht und dabei geholfen werden, aus ihnen zu lernen.

\section{Material und Methoden}

$\nabla$

Die vorliegende Untersuchung basiert auf Datenmaterial der SST. Untersucht wurden 88 abgeschlossene Fälle der SST zu Mammareduktionen aus dem Zeitraum der Jahre 2000-2007. Der länger zurück liegende Zeitraum ist damit begründet, dass die Fälle erst dann abgeschlossen werden konnten, wenn kein Gerichtsverfahren angestrengt wurde. Dafür hat die Schlichtungsstelle einen Zeitraum von 5 Jahren vorgesehen.

Bei der SST erfolgt die Archivierung abgeschlossener Schlichtungsfälle anhand des MERS. Für die vorliegende Untersuchung wurden anhand der Erfassung des MERS die Fälle mit den aufgeführten ICD-10 Klassifikationen für Brustoperationen zusammengestellt und in Form von Excel-Datenblättern sowie den Akten selbst zur Verfügung gestellt.

Alle Fälle, in denen die Kausalität eines ärztlichen Behandlungsfehlers mit einem daraus folgenden Schaden bejaht und damit ein Haftungsanspruch bestätigt wurde, wurden genauer analysiert.

\section{Ergebnisse}

Unter den 88 in der SST bearbeiteten Mammareduktionen wurden der Großteil stationär, aber immerhin 5 Patientinnen ambulant operiert. Es fanden sich 37 Eingriffe mit Haftungsanspruch ( $\bullet$ Tab. 1), bei denen in 3 Fällen aus Sicht der Gutachter keine Operationsindikation bestanden hatte. Es waren 10 Fälle durch einen Allgemeinchirurgen operiert worden, davon 7 mit Haftungsanspruch, 26 Fälle durch einen Plastischen Chirurgen, davon 9 mit Haftungsanspruch und 49 Fälle durch einen Gynäkologen, davon 21 mit Haftungsanspruch. Ein statistisch aussagekräftiger Vergleich zwischen den jeweiligen Fachgruppen ist allerdings bei diesen Fallzahlen nicht möglich.

Es wurde entweder zu viel oder zu wenig Gewebe entnommen oder die Mamillendurchblutung nicht beachtet. Fehlerquelle für eine Nekrose des Mamillenareolar-Komplexes (MAK) waren entweder eine intraoperativ mangelhafte Schnittführung mit ungenügender Beachtung der Mamillendurchblutung oder eine zu späte Reaktion auf postoperativ aufgetretene Durchblutungsstörungen und Hämatome. In $21 \%$ der Mammareduktionen erkannte die Schlichtungsstelle eine Nekrose des MAK als fehlerbedingten Schaden an.

Speziell die OP-Planung wurde hier in $65 \%$ der Fälle bemängelt. In 7 Fällen bemängelten die Gutachter, dass das Ziel der Operation nicht erreicht worden sei, und in 4 Fällen, dass die zeitliche
Tab. 1 Mängel bei der Operation.

\begin{tabular}{|lc}
\hline Mängel & Anzahl (Prozent) \\
\hline Mängel erwähnt & $34(92 \%)$ \\
\hline Keine Mängel erwähnt & $3(8 \%)$ \\
Mängel genauer beschrieben (Mehrfachnennung möglich): \\
\hline OP-Planung & $24(65 \%)$ \\
Schnittführung & $15(41 \%)$ \\
\hline Revisionsoperation fehlerhaft & $10(27 \%)$ \\
Weitere & $9(24 \%)$
\end{tabular}

\section{Tab. 2 Mängel OP-Planung.}

\begin{tabular}{|ll|}
\hline Mängel OP-Planung & Anzahl \\
\hline Falsche Methode & 12 \\
\hline Methode wurde falsch abgewandelt & 3 \\
\hline Zeitliche Planung fehlerhaft & 4 \\
\hline OP-Ziel wurde nicht erreicht & 7 \\
\hline
\end{tabular}

Tab. 3 Mängel Schnittführung.

\section{Mängel Schnittführung}

Mamillendurchblutung wurde nicht beachtet

Zu viel entnommen

Zu wenig entnommen

Anzahl
8
3
5

Planung (z.B. ein mehrzeitiges Vorgehen) nicht korrekt gewesen sei. In 12 der Fälle wurde im Gutachten angegeben, dass die falsche OP-Methode gewählt, und in 3 Fällen, dass die OP-Methode falsch abgewandelt worden sei ( $\bullet$ Tab. 2). Hinsichtlich der Planung wurden entweder der zeitliche Faktor, z.B. der Zeitpunkt einer Revisionsoperation als fehlerhaft bewertet. In einem anderen Fall wurde die Brustreduktion vor einem von einer Patientin angestrebtem Gewichtsverlust, bemängelt. Durch nachträglichen Gewichtsverlust reduzierte sich das Brustvolumen der Patientin erneut, letztendlich wurde eine Brustaugmentation zum Volumenausgleich nötig. Dem Operateur war der Wunsch der Gewichtsreduktion vor der initialen OP bekannt. In der Aufklärung wurde zudem nicht explizit auf einen möglichen Volumenverlust durch Gewichtsveränderungen hingewiesen.

Die Bewertung der OP-Planung wurde naturgemäß von den Gutachtern nach Aktenlage gemacht. Hier spielten die Aufklärung z.B. über die OP Methoden, die präoperativen Untersuchungsbefunde, z.B. präoperative Abmessungen sowie prä- und postoperative Fotodokumentationen entscheidende Rollen. Die genannten Faktoren fanden sich wiederholt lückenhaft in den Akten.

Auch die OP-Nachsorge war häufig unzureichend nachvollziehbar dokumentiert, hier wurden z. B. Ultraschalluntersuchungen bei Hämatombildung, Verbands- und Wundkontrollen nicht angemessen dokumentiert. Zudem fielen wiederholt die sehr kurzen OP-Berichte mit z.B. fehlenden Angaben zur Mammillendurchblutung auf.

In diesen Fällen führte die mangelhafte Dokumentation und Nachvollziehbarkeit von Behandlungsabläufen zu einer Beweislastumkehr zu Lasten des Arztes.

Auch die Schnittführung und das Resektionsgewicht wurden häufig von den Gutachtern bemängelt ( $\odot$ Tab. 3).

25 Patientinnen klagten über postoperativ asymmetrische bzw. deformierte Brüste. In 9 Fällen wurde die Nekrose bzw. der Verlust des MAK beklagt, in 8 Fällen war die Brustgröße Gegenstand von Beschwerden und in 6 Fällen Wundinfektionen, ( $\bullet$ Abb. 1$)$. 


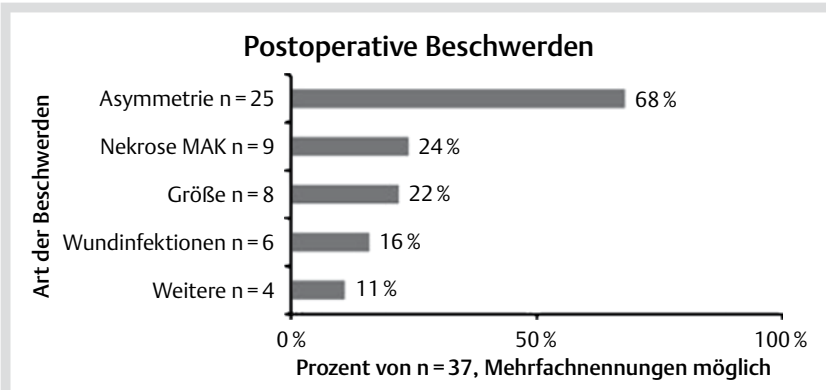

Abb. 1 Postoperative Beschwerden.

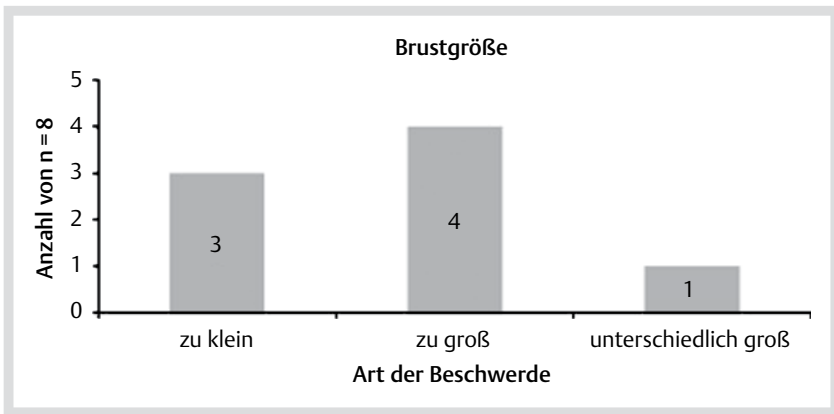

Abb. 2 Art der Beschwerde.

Tab. 4 Mängel bei Planung+ Durchführung.

$\begin{array}{ll}\text { Weitere Mängel bei der Planung oder Durchführung } & \text { Anzahl } \\ \text { Keine Reaktion auf Hämatome } & 3 \\ \begin{array}{l}\text { Grobe Dokumentationsfehler, sodass die } \\ \text { Operationsdurchführung nicht nachvollziehbar ist }\end{array} & 3 \\ \text { Falsche Nahttechnik } & 1 \\ \text { Tupfer hinterlassen } & 2\end{array}$

Innerhalb der 8 Fälle mit Beschwerden über die Brustgröße wurde 4-mal beklagt, die Brüste seien weiterhin zu groß, 3-mal sie seien zu klein und einmal wurde die unterschiedliche Größe kritisiert $\bullet$ Abb. 2. Weitere Mängel finden sich in $\odot$ Tab. 4.

Die $\diamond$ Tab. 5 fasst noch einmal die häufigsten Beschwerdepunkte, die die Patientinnen nach den Behandlungen erwähnten, zusammen. Die 2 häufigsten Punkte betrafen Asymmetrien und Deformitäten der postoperativen Brust sowie die Narbenbildung.

Narben treten naturgemäß durch eine Operation auf, sie werden durch Patienten aber häufig als eine Komplikation einer Operation gedeutet. In den Antragsschreiben der Schlichtungsverfahren beklagen in den FMH 78\% der Patientinnen die entstandenen Narben. O Tab. 6 listet die Beschwerden über Narben auf, $\odot$ Tab. 7 liefert Informationen aus Revisionsoperationen.

Auch wenn Revisionsoperationen indiziert worden waren, ergab sich hier eine Fehlerquelle. In 27\% der FMH bei Mammareduktion wurde auch die Durchführung und Planung der Revisionsoperation als fehlerhaft bewertet. In dem durch die SST auf Grundlage der Gutachten erarbeitetem Schlichtungsbescheid wird dazu Stellung genommen, welche Schäden als fehlerbedingt eingestuft wurden ( $\bullet$ Tab. 8 )

Die Nekrose des MAK wurde in $22 \%$ aller Fälle als Schaden gesehen, die erneute Operation an sich war in je ca. 50\% der Fälle der häufigste Schaden überhaupt. Ähnlich häufig fand sich das schlechte ästhetische Ergebnis sowie die entstandenen Narben (22\%).
Tab. 5 Beschwerden der Patientinnen.

\begin{tabular}{|lr|}
\hline Häufigsten Beschwerden & Reduktionen $\mathbf{n = 3 7}$ \\
\hline Brustgröße & $8(22 \%)$ \\
\hline Asymmetrie/Deformität der Brüste & $25(68 \%)$ \\
\hline Wundinfektionen & $6(16 \%)$ \\
\hline Narben & $29(78 \%)$ \\
\hline Behandlungsnachsorge & $19(51 \%)$ \\
\hline Finanzielle Schäden & $25(46 \%)$ \\
\hline Psychologische Belastung & $26(70 \%)$ \\
\hline Risikoaufklärung & $12(32 \%)$ \\
\hline
\end{tabular}

Tab. 6 Beschwerden über Narben.

\begin{tabular}{lc} 
Beschwerden Narben & $\begin{array}{c}\text { Anzahl (Prozent) } \\
\mathbf{n = 3 7} \text { Reduktionen }\end{array}$ \\
\hline Nicht erwähnt & $8(28 \%)$ \\
\hline erwähnt & $29(78 \%)$ \\
Wenn Mängel hinsichtlich Operationsnarben erwähnt wurden, was \\
wurde bemängelt? \\
Lage \\
Größe & $7(19 \%)$ \\
\hline Länge & $15(41 \%)$ \\
Wundheilungsstörung & $1(3 \%)$ \\
\hline Weitere & $18(49 \%)$ \\
\hline
\end{tabular}

Tab. 7 Revisionsoperationen.

\begin{tabular}{|l|c|}
\hline Anzahl der Revisionsoperationen & Reduktion $\mathbf{n = 3 7}$ \\
\hline keine Revisionsoperation & $10(27 \%)$ \\
\hline 1 Revision oder Revision ist noch nötig & $14(38 \%)$ \\
\hline 2 Revisionsoperationen & $4(11 \%)$ \\
\hline 3 Revisionsoperationen & $6(16 \%)$ \\
\hline mehr als 3 Revisionsoperationen & $3(8 \%)$ \\
\hline
\end{tabular}

Tab. 8 Schaden.

\begin{tabular}{lc} 
Schaden & $\begin{array}{c}\text { Reduktion } \\
\mathbf{n = 3 7}\end{array}$ \\
Nicht genau erwähnt & $0(0 \%)$ \\
\hline Erwähnt & $37(100 \%)$ \\
Schäden genauer: & \\
Erneute Operation & $20(54 \%)$ \\
\hline Psychologische Belastung & $12(32 \%)$ \\
\hline Narben & $8(22 \%)$ \\
\hline Schlechtes kosmetisches Ergebnis & $15(41 \%)$ \\
Nachsorgemangel & $3(8 \%)$ \\
\hline Asymmetrie der Brüste & $7(19 \%)$ \\
Plexuslähmung durch fehlerhafte Lagerung & $2(5 \%)$ \\
\hline Nekrose des MAK & $8(22 \%)$
\end{tabular}

Häufig wurden Narbenbildungen gerügt, welche im Rahmen von vermeidbaren Wundheilungsstörungen, wie z.B. durch Nichtbeachtung der Mammillendurchblutung oder zu späte Reaktion auf Hämatome, aufgetreten waren. Ein Arzt spülte die Wunden z.B. aber auch mit einer Persillauge. Auch wurde in einem Fall die Mammille mit Einzelknopfnähten statt subcutaner Naht vernäht, daraus resultierte ein sternförmiges Narbenbild um die Mammille.

Die Rüge über das schlechte ästhetische Ergebnis ergab sich größtenteils aus der Summation verschiedener mangelhafter 
Tab. 9 Vermeidbare Fehler.

\begin{tabular}{ll} 
Vermeidbare Fehler aus Sicht des Gutachters & \\
Freie Mamillentransposition wäre besser gewesen & $2(7 \%)$ \\
\hline Keine Beachtung der MAK Durchblutung & $7(19 \%)$ \\
Falscher Umgang mit den Komplikationen & $7(19 \%)$ \\
\hline $\begin{array}{l}\text { Andere Methode zur Reduktion wäre besser gewesen } \\
\text { bzw. Methode wurde falsch abgewandelt }\end{array}$ & $6(16 \%)$ \\
Zu wenig Brustgewebe entnommen & $4(11 \%)$
\end{tabular}

Behandlungsschritte, z.B. ungleich angelegte Submammärfalten, unterschiedlich hoch positionierte MAKs, massive Wundheilungsstörungen, der teilweise oder ganze Verlust des Brustdrüsengewebes durch z.B. die Veränderung von etablierten OP-Verfahren und daraus resultierender Zerstörung der Mammillendurchblutung.

Es wurde wiederholt die fehlende Aufklärung über alternative OP-Methoden gerügt, z.B. eine freie Mammillentransposition bei Gigantomastie oder dass eine OP mit einer Schnittführung durchgeführt wurde, über welche vorab nicht aufgeklärt wurde. Hier resultierte u.a. ein anderes Narbenbild als besprochen. Als psychologische Belastungen wurden verlängerte Behandlungszeiträume bis hin zum gestörten Körperverhältnis der Frauen postoperativ durch z.B. Verlust der Mammillen gezählt.

Aus Sicht der Gutachter wären unter Berücksichtigung der anatomischen und operationstechnischen Bedingungen einige Fehler vermeidbar gewesen ( $\bullet$ Tab. 9).

\section{Fallbeispiel}

Deutlich wird ein OP-Planungsfehler sowie Fehler in der Schnittführung, aber auch eine fehlerhafte bedingte Narbenbildung und psychologisch Belastung in folgendem

\section{Schlichtungsfall}

Eine Patientin wünschte bei extremer Makromastie eine Brustreduktion. Ihr behandelnder Arzt entschied sich zu einer Reduktionsplastik mit inferiorem Mammillenstiel. Die erste Operation verlief bei großem Resektionsgewicht komplikationslos. Im postoperativen Verlauf kam es zu einem Dog-Ear. Die Patientin wünschte daraufhin eine Narbenkorrektur und ließ sich erneut vom gleichen Arzt behandeln. Dieser führte die OP mit dem Ziel der Narbenkorrektur durch. In dieser OP erfolgte eine erneute Gewebsreduktion. Diese war mit der Patientin jedoch nicht vereinbart worden und nicht gewünscht, da sie ihre Brüste schon als recht klein empfand. Der Operateur versäumte zudem, die durch ihn geschaffene inferiore Mamillenstielung zu erhalten, indem er sie im Rahmen der erneuten Gewebsreduktion durchtrennte. Eine wirkliche Narbenkorrektur erfolgte nicht. Im weiteren Verlauf traten Wundheilungsstörungen mit Nekrosen und Verlust des MAK und des verbliebenen Brustdrüsengewebes auf. Es folgten 3 weitere Operationen wegen der Wundheilungsstörungen, das Narbenbild verschlimmerte sich und es kam zu einem fast vollständige Verlust des Brustdrüsengewebes. Zudem beklagte die Patientin finanzielle Verluste und psychologische Probleme. Im Schlichtungbescheid heißt es, dass zum einem für die erneute Gewebsreduktion keine Indikation bestand und zum anderen dem Operateur ein grober Planungsfehler sowie Fehler in der Schnittführung unterliefen. Da er beide Eingriffe selbst durchgeführt hat, hätte er in der zweiten OP wissen müssen, wie er die MAK-Durchblutung korrekt erhält. Der Planungsfehler liegt in der Revisionsoperation. Die Wahl der OP-Methode war nicht geeignet, um das eigentliche Problem, Dog-Ear, zu beheben.
Durch die Behandlung sei zudem eine passagere Depression aufgetreten.

\section{Diskussion \\ $\nabla$}

Innerhalb der Jahre 2004-2008 wurden durch Schlichtungsstellen in Deutschland rund 13300 Entscheidungen getroffen. In insgesamt 27\% wurden die Kausalität zwischen Behandlungsfehler und dem beklagten Schaden bestätigt, sodass sich hier ein Haftungsanspruch ergab [6]. Gille und Neu konnten 2012 nachweisen, dass in operativen Fächern der häufigste Fehler bei der Durchführung der Operation entsteht. In der Diagnostik betreffen die meisten Fehler die bildgebenden Verfahren [10].

Die Schlichtungsfälle verdeutlichen die Komplexität der Fehlermöglichkeiten in der OP-Planung und -Durchführung. Um Fehlerquoten möglichst gering zu halten, ist eine genaueste OP-Planung unabdingbar. Diese muss anhand der anatomischen und pathologischen Bedingungen getroffen werden und setzt sehr gute Kenntnisse der verschiedenen Operationstechniken und Risiken voraus. Der behandelnde Arzt sollte sich - auch das bestätigen die in die Studie eingegangenen Fälle - niemals nur von den Wünschen einer Patientin hinsichtlich einer bestimmten Operationsmethode leiten lassen. Auch wenn die Mammareduktion häufig eine Wunschoperation ist, begründet der Wunsch alleine nicht die Indikation bzw. Rechtfertigung zur Operation oder Wahl der OP-Methode.

Es handelt sich bei Mammareduktionen um eine Behandlung, welche mit einer hohen Erwartungshaltung der Patientinnen an das Ergebnis und an die Leistung des Arztes einhergeht. Um die hohen Qualitätsansprüche, welche an plastisch-chirurgische Operationen gestellt werden, zu erfüllen, ist laut Vogt ein intensives Risikomanagement intra- sowie postoperativ gefragt. Ein korrektes Komplikationsmanagement sollte präoperativ mit einer sorgfältigen Patientenauswahl und einer genauen Indikationsstellung beginnen, das Ergebnis sollte postoperativ durch eine stetige Nachsorge gesichert werden [11]. Die Aufgabe des Operateurs ist es zudem, sich die Frage zu stellen, ob er dem Wunsch der Patientin gerecht werden kann, ob der Eingriff dem Wohl der Patientin dient und er ihr nicht schadet [12].

Die Mammareduktionsplastik ist ein etablierter Eingriff, der zumeist wegen funktioneller Rücken- und Nackenschmerzen oder auch einem nachvollziehbaren, psychosozialen Leidendruck durchgeführt wird $[13,14]$ Insgesamt hat die Operation einen positiven Effekt auf die Lebensqualität der Frauen $[13,15,16]$. Dennoch klagen die Patientinnen jedoch nicht selten vor Gerichten auf Schadensersatz oder beantragen ein Schlichtungsverfahren bei Gutachterkommissionen und Schlichtungsstellen bei Komplikationen wie Wundheilungsstörungen oder Nekrosen der Mamillen. Denn Komplikationen können zu schlechten ästhetischen Ergebnissen führen, welche zudem ggf. kaum bis schwer korrigierbar sein können [12].

Bei den 37 vorliegenden Fällen wurden in 92\% Mängel in der OP-Planung und -Durchführung erwähnt. An erster Stelle steht die OP-Planung, die in 65\% der FMH als mangelhaft bewertet wurde.

Mit einer Häufigkeit von 10-53\% werden Komplikationen bei Mammareduktionen in der Literatur angegeben $[11,17,18]$ In einer retrospektiven Studie von Shah et al. mit 306 Fällen der Mammareduktion betrug die Gesamtrate an Komplikationen $53,9 \%$ [18]. Am häufigsten traten Wunddehiszenzen (26,5\%) auf. 
Rauchen, große Resektionsmenge und freie Mamillentransplantation waren in dieser Studie assoziiert mit einer erhöhten Komplikationsrate.

84\% der Patientinnen mit FMH, welche sich einer Mammareduktion unterzogen hatten, waren nicht mit dem postoperativen Ergebnis zufrieden. Hauptbeschwerden waren entstandene Asymmetrien und Deformitäten der Brüste (68\%). 24\% beklagten eine Nekrose des MAK, 22\% die Brustgröße, 16\% hatten mit Wundinfektionen zu tun.

Menke et al. berichteten über eine Standardtechnik zur Brustreduktion mit vertikaler Narbe an über 800 Patientinnen als narbenschonende Alternative zu den T-Schnitt Techniken. Genaue Komplikationsraten finden sich in der Arbeit jedoch nicht [19]. Weiterhin findet sich in der deutschen Literatur eine Arbeit von Kneser et al., worin von 814 mit einer Mammareduktion behandelten Patientinnen 161 per Fragebogen nachuntersucht wurden. Hiervon beschrieben $47 \%$ die postoperativen Narben als „mäßig breit“ und 16\% als „störend breit“ und $12 \%$ beklagten schmerzhafte Narben [15]. Ferner empfanden nur 60\% die Form postoperativ als symmetrisch [15]. Auch in der Arbeit von Horch et al. finden sich bei $13 \%$ von 223 Patientinnen, welche per Fragebogen nachuntersucht worden sind, postoperative Narbenbeschwerden. In diesem Patientenkollektiv empfanden $16 \%$ der Frauen ihre Brustgröße auch nach der Operation als noch zu groß [13].

In der internationalen Literatur liegen die publizierten Komplikationsraten gelegentlich höher. In der Arbeit von Setälä fanden sich bei 273 Mammareduktionen eine Komplikationsrate von $52 \%$. Die häufigsten Komplikationen waren eine verzögerte Wundheilung wegen oberflächlicher Infektionen (26\%), Serome (8\%), Hautnekrosen oder Wunddehiszenz (18\%) und tiefe Infektionen (8\%). Bei übergewichtigen Patientinnen war die Nekrose des MAK häufiger als bei normalgewichtigen Patientinnen (6\% vs $0 \%)$. Ebenso waren das Resektionsgewicht und der ursprüngliche Abstand zwischen Klavikula und MAK mit einem erhöhten Risiko für MAK-Nekrosen assoziiert [17]. 23\% der Patientinnen wurden erneut operiert, am häufigsten wegen Wundheilungsstörungen $(8,8 \%)$, Blutungen (4\%), tiefen Infektionen $(1,1 \%)$, Narben oder Falten (13\%). Die aufgetretenen Komplikationen im untersuchten Patientengut mit FMH lassen keine Rückschlüsse auf allgemeine Komplikationsraten ziehen. Sie sind aber vergleichbar zu den in der Literatur beschriebenen Komplikationen bei Mammareduktionen und bieten einen guten Überblick darüber, was Patientinnen nach einer Operation als störend empfinden.

Aus den Schlichtungsurteilen wurden bei den $37 \mathrm{FMH}$ bei Mammareduktionen ähnliche Punkte als fehlerbedingte Schäden eingestuft. Dieses waren insbesondere erneute Operationen (54\%), das schlechte ästhetische Ergebnis (41\%), psychologische Belastungen (32\%), Narben (22\%), Nekrosen des MAK (22\%) und Asymmetrien der Brüste (19\%).

Da es bei der subjektiven Brustvermessung in der Regel zu Fehlbestimmungen des Volumens kommt, wobei kleinere Brüste als zu groß und große Brüste als zu klein bestimmt werden [20], bietet neuerdings die Messung mit einer „Breast Analysis Tool“ (BAT) Software eine höhere Objektivität und somit die Möglichkeit, subjektiv von den Patienten empfundene Asymmetrien und/oder möglicherweise verfehlte Volumenreduktionen zu objektivieren.

Insgesamt bietet das Datenmaterial der Schlichtungsstelle einen guten Einblick in mögliche Fehlerquellen bei der Mammareduktion. Es lehrt uns, dass Fehler auf allen Ebenen einer Behandlung entstehen. Viele werden bereits bei der Planung schon vor Be- ginn der eigentlichen Operation eingeleitet. Formverändernde ästhetische Brustoperationen sind somit komplexe Behandlungen, welche eine gute theoretische und praktische Ausbildung der Operateure voraussetzen, damit diese in allen Schritten der Behandlung die richtigen Entscheidungen für ein erfolgreiches Behandlungsergebnis treffen können.

Eine grundlegende Schwachstelle der Schlichtungsverfahren ist jedoch, dass die Gutachter ihre Gutachten naturgemäß ex post nach Aktenlage und zumeist nach persönlicher Untersuchung der Kläger erstellen. Die tatsächlichen Abläufe der beklagten Operationen, einschließlich der vorausgegangenen Indikationstellungen und Planungen sowie Nachbehandlungen sollten daher sehr genau dokumentiert werden. Aus den hier vorliegenden Daten können entscheidende Punkte zur Fehlervermeidung abgelesen werden.

Neben der korrekten Aufklärung [21] ist die korrekte Anzeichnung im Stehen bei wacher Patientin, das intraoperative Überprüfen dieser Anzeichnung, das wiederholte Aufsetzen der Patientin im Operationssaal zur Überprüfung der Zwischen- und Endergebnisse der Operationen essentiell [22]. Ferner ist natürlich eine präzise und korrekte Dokumentation der OP Planung (Brustvermessungsbogen) ebenso wie der Operationsschritte notwendig. Die korrekte Dokumentation sollte neben der rein

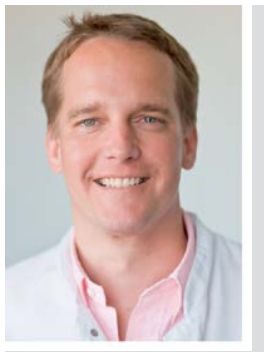

Sixtus Allert Nach Studium in Bonn, Wien und New Jersey zunächst allgemein- und unfallchirurgische Ausbildung an der Klinik der Ludwig-Maximilians-Universität in München (Prof. Dr. L. Schweiberer). Weiterbildung zum Plastischen Chirurgen an der Klinik für Plastische, Hand- und Wiederherstellungschirurgie der Medizinischen Hochschule Hannover mit Facharztanerkennung für Plastische Chirurgie (Prof. Dr. A. Berger). Nach Stationen in Hameln und Düsseldorf Zusatzbezeichnung Handchirurgie am Friederikenstift Hannover (Frau Prof. Dr. M. Flügel). Seit 2006 Chefarzt der Klinik für Plastische und Ästhetische Chirurgie/ Handchirurgie des Sana Klinikums Hameln-Pyrmont, seit 2009 Ärztlicher Direktor des Klinikums. Mitglied für Plastische Chirurgie der Norddeutschen Schlichtungsstelle.

handwerklichen operativen Ausbildung in der Chirurgie ein wichtiger Bestandteil sein. Die Ansprüche an eine ordnungsgemäße Aufklärung steigen stetig.

\section{Interessenkonflikt: Nein}

\section{Literatur}

1 Kohn L, Corrigan J, Donaldson MS. To Err Is Human: Building a Safer Health System. Washington, DC: Committee on Quality of Health Care in America, Institute of Medicine; 2000

2 Schaffartzik W, Neu J. Definition des ärztlichen Behandlungsfehlers. Trauma und Berufskrankheit 2008; 10: 196-199

3 Hansis $M$, Hart $D$, Becker $K$. Medizinische Behandlungsfehler in Deutschland. Gesundheitsberichterstattung des Bundes, Institut RK; Berlin: 2001

4 Neu J. Das Verfahren der Norddeutschen Schlichtungsstelle. Zeitschrift für Herz-,Thorax- und Gefäßchirurgie 2009; 23: 93-96

5 http://www.norddeutsche-schlichtungsstelle.de/download am 15.12 .15

$6 \mathrm{Neu}$ J. Arbeit und Ergebnisse der Schlichtungsstellen in Deutschland - am Beispiel der Norddeutschen Schlichtungsstelle. Der Chirurg BDC 2009; 6: 314-318

$7 \mathrm{Neu}$ J. Das Verfahren der Norddeutschen Schlichtungsstelle. Zeitschrift für Herz-,Thorax- und Gefäßchirurgie 2009; 23: 93-96 
8 Feldkamp A. Schlichten statt richten - Fehler in der Medizin. Klinikarzt 2010; 39: 380-381

$9 \mathrm{Neu} \mathrm{J}$. Das Verfahren der Norddeutschen. Schlichtungsstelle

10 Gille J, Neu J. Erfahrungen der ärztlichen Schlichtungsstelle. Gynäkologische Praxis 2012; 36: 203-212

11 Vogt P. Postoperative Komplikationen in der plastischen Chirurgie. Der Chirurg 2009; 80: 827-839

12 Nestle-Krämling C, Beck L. Ästhetische Chirurgie in der Gynäkologie. Der Gynäkologe 2007; 40: 92-98

13 Horch RE, Jaeger K, Stark GB. Quality of life after breast reduction-plasty. Handchir Mikrochir Plast Chir 1999; 31: 137-142

14 Scholz T, Diedrichson J, Olbrisch RR et al. Cost analysis of conservative versus operative therapy of macromastia. Handchir Mikrochir Plast Chir 2008; 40: 100-104

15 Kneser U, Jaeger K, Bach AD et al. Breast-reduction surgery - a longterm survey of indications and outcomes. MMW Fortschr Med 2004; 146: 36-38 40

16 Spector JA, Karp NS. Reduction mammaplasty: a significant improvement at any size. Plast Reconstr Surg 2007; 120: 845-850
17 Setala L, Papp A, Joukainen S et al. Obesity and complications in breast reduction surgery: are restrictions justified? J Plast Reconstr Aesthet Surg 2009; 62: 195-199

18 Shah R, Al-Ajam Y, Stott D et al. Obesity in mammaplasty: a study of complications following breast reduction. J Plast Reconstr Aesthet Surg 2011; 64: 508-514

19 Menke H, Olbrisch RR, Bahr C. Standard technique of breast reduction surgery with vertical scar. Handchir Mikrochir Plast Chir 1999; 31: 134-136

20 Henseler H, Hille-Betz U, Vogt PM. Validation of Subjective Estimates of Female Breast Volume and Comparison with Objective Methods. Handchir Mikrochir Plast Chir 2015; 47: 371-377

21 Allert S. Aufklärung vor ästhetisch-chirurgischen Eingriffen und Dokumentation. In: von Heimburg, Lemoperle, Richter - Ästhetische Chirurgie, Kap. XIV -2b 30. Erg. Lfg. 12/13 Seiten 1-4

22 Allert S. Spezielle Fragen der Begutachtung in der Ästhetischen Chirurgie. In: von Heimburg, Lemperle, Richter - Ästhetische Chirurgie, Kap. XIV -5b 30. Erg. Lfg. 12/13 Seiten 1-2 